\title{
EVALUACIÓN DE LA CALIDAD DEL DESTILADO ALCOHÓLICO DE ANÍS VERDE (Pimpinella anisum L.) OBTENIDO POR DESTILACIÓN FRACCIONADA
}

\author{
Clesez Tunqui Quispe ${ }^{* a}$, Alexia Pardo Figueroa Dianderas ${ }^{\mathrm{b}}$, Gustavo Tejada Flores ${ }^{\mathrm{b}}$, \\ Ivette del Rosario Cjuro Farfán ${ }^{c}$
}

\begin{abstract}
RESUMEN
En este trabajo de investigación se evaluó la calidad del destilado alcohólico de anís verde (Pimpinella anisum L.) obtenido por destilación fraccionada en una columna de uno (01), dos (02) y tres (03) platos; el cual es un producto intermedio para la elaboración de anisado. El proceso productivo contempló la mezcla de aguardiente y granos de anís verde para su posterior maceración, una vez obtenido el macerado, se procedió a su destilación en un alambique de columna fraccionada. La metodología para la evaluación consistió en la realización de análisis organoléptico, fisicoquímicos, espectroscópicos (UV-visible) y cromatográficos (CCF, CLAE y CG/EM). Se realizaron cinco (05) corridas de producción para obtener cinco (05) muestras de destilado por cada tamaño de columna (uno, dos y tres platos). En general, el destilado alcohólico de anís verde se caracterizó por la elevada presencia de compuestos carbonílicos, y moderada presencia de compuestos insaturados y carbohidratos. Los análisis de CCF, CLAE y CG/EM evidencian la presencia de trans-Anetol. En función a sus características se concluyó que el mejor destilado para la formulación de anisados es el obtenido en la destilación con dos (02) platos.
\end{abstract}

Palabras clave: anís verde (P. anisum L.), destilado alcohólico, destilación fraccionada, trans-Anetol.

\section{ASSESSMENT OF THE QUALITY OF THE GREEN ANISE (PIMPINELLA ANISUM L.) ALCOHOLIC DISTILLATE OBTAINED BY FRACTIONAL DISTILLATION}

\begin{abstract}
In this research work, it was evaluated the quality of the green anise (P. anisum L.) alcoholic distillate obtained by fractional distillation in a column of one, two and three plates; which is an intermediate product for the production of aniseed. The production process involved the

\footnotetext{
${ }^{* a}$ Facultad de Química, Universidad Nacional de San Agustín, Calle Santa Catalina № 117, Arequipa, Perú, cleseztq1402@gmail.com

b Facultad de Ingeniería y Computación, Universidad Católica San Pablo, Quinta Vivanco S/N Urb. Campiña Paisajista, Arequipa, Perú.

c Facultad de Ingeniería de Procesos, Universidad Nacional de San Agustín, Calle Santa Catalina N ${ }^{\circ} 117$, Arequipa, Perú.
} 
mixture of schnapps and green anise grains for subsequent maceration. The macerated was distilled in a fractional column pot still. The evaluation methodology included organoleptic, physicochemical, spectroscopic (UV-visible) and chromatographic (TLC, HPLC and GC/ MS) analysis. In order to obtain five samples for each column size (one, two and three plates), five production runs were carried. In general, the alcoholic distillate of green anise had characterized by the high presence of carbonyl compounds, and the moderate presence of unsaturated compounds and carbohydrates. The analysis of TLC, HPLC and GC/MS demonstrate the presence of trans-Anethole. Based on its characteristics, it was concluded that the best distillate for the production of aniseed is obtained in the distillation with two (02) plates.

Key words: green anise (P. anisum L.), alcoholic distillate, fractional distillation, transAnethole.

\section{INTRODUCCIÓN}

El anís ( P. anisum L.) es una planta herbácea muy aromática; su aceite esencial se utiliza como aditivo, aromatizante y saborizante en las industrias alimentaria, farmacéutica, cosmética, entre otras ${ }^{1}$. En estudios referentes al anís verde, se ha demostrado que su aceite esencial posee una composición variable de metabolitos secundarios según la zona de la que proviene, el tiempo de cosecha y el clima; metabolitos de los cuales los más importantes son el transAnetol y el $p$-Anisaldehído ${ }^{2}$. Se ha evidenciado que no existen estudios, investigaciones ni información específicamente sobre la caracterización del destilado alcohólico de anís verde obtenido por destilación fraccionada, sin embargo, existen trabajos de investigación que describen y determinan las características del aceite esencial de anís verde ${ }^{2,3,4}$. La empresa donde se realizó la investigación actualmente produce anisados a base de destilado alcohólico de anís verde ( $P$. anisum L.), pero no ha realizado estudios sobre las principales características y componentes del destilado obtenido por destilación fraccionada en columna de uno (01), dos (02) y tres (03) platos. Por esta razón, el objetivo de la investigación fue evaluar las propiedades de los tres (03) destilados alcohólicos de anís verde (P. anisum L.) e identificar, según la evaluación de sus características, cuál de los tres (03) es el mejor para formular anisados.

\section{PARTE EXPERIMENTAL}

La investigación se realizó en el Laboratorio de Química Orgánica de la Universidad Nacional de San Agustín (Arequipa, Perú), Laboratorio de Control de Calidad de la Universidad Católica de Santa María (Arequipa, Perú) e instalaciones de una industria licorera productora de anisados (Arequipa, Perú).

\section{Identificación taxonómica del anís verde}

En la tabla 1 se muestra los resultados de la identificación taxonómica del anís verde. 
Tabla 1. Identificación taxonómica del anís verde.

\begin{tabular}{llclll}
\hline \multicolumn{5}{c}{ Clasificación científica } \\
\hline Reino & Plantae & División & Magnoliophyta & Clase & Magnoliopsida \\
Subclase & Rosidae & Orden & Apiales & Familia & Apiaceae \\
Subfamilia & Apiodeae & Género & Pimpinella & Especie & P. anisum L. \\
\hline
\end{tabular}

Fuente: Herbarium Areqvipense (HUSA) Universidad Nacional de San Agustín.

El ejemplar de la especie en estudio (P. anisum L.) fue depositado e identificado en el herbario "Herbarium Areqvipense (HUSA) de la Universidad Nacional de San Agustín.

\section{Muestreo y preparación de las muestras}

Para que las muestras sean representativas, se controlaron y estandarizaron los siguientes parámetros: masa de anís verde, grado alcohólico y masa del aguardiente, tiempo de maceración, y masa del macerado que se carga al alambique. El muestreo se realizó en una empresa licorera, específicamente en el área de producción del destilado de anís.

Se inició el proceso productivo con la formulación y masado de 115,85 kg de aguardiente, masado de 12,60 kg de anís verde, y maceración por 15 días en tanques de acero inoxidable de 200 L de capacidad. Luego, se transportó el macerado a la olla de cocción de cobre del alambique de columna fraccionada (propiedad de la industria licorera en la que se realizó el trabajo) con tres (03) platos para su destilación, proceso que se repitió cinco (05) veces para obtener las muestras. Se siguió el mismo proceso para el muestreo de destilado obtenido en un alambique de dos (02) y de un (01) plato. El alambique posee un sistema clamp que permite retirar y colocar platos de destilación según el número de platos con el que se desee trabajar. Las muestras fueron tomadas en quince (15) corridas de producción (05 por cada tamaño de columna), todas por un volumen de $500 \mathrm{~mL}$ y correctamente rotuladas para su análisis por triplicado. El muestreo se realizó basándose en la NTP 319.079.1974.

\section{Análisis organoléptico}

Las muestras fueron analizadas por personas con experiencia en cata de bebidas alcohólicas (trabajan en la industria licorera productora de anisados) siguiendo las normas NTP-ISO 11035:20097 y NCh107 y los datos colectados se muestran en la tabla 2.

Tabla 2. Ponderaciones para el análisis organoléptico de destilado de anís verde.

\begin{tabular}{|c|c|c|c|c|}
\hline Nivel & Sabor & Color & Olor & $\begin{array}{c}\text { Estado } \\
\text { físico }\end{array}$ \\
\hline 1 & Ácido y picante & $\begin{array}{c}\text { Café oscuro, } \\
\text { amarillento y turbio }\end{array}$ & $\begin{array}{l}\text { Etanólico, ligeramente } \\
\text { aromático a anís }\end{array}$ & $\begin{array}{c}\text { Líquido } \\
\text { opalescente }\end{array}$ \\
\hline 2 & $\begin{array}{l}\text { Intensamente ácido, } \\
\text { ligeramente dulce }\end{array}$ & $\begin{array}{l}\text { Incoloro, } \\
\text { ligeramente } \\
\text { amarillento }\end{array}$ & $\begin{array}{l}\text { Ligeramente aromático } \\
\text { a anís, penetrante }\end{array}$ & $\begin{array}{l}\text { Líquido } \\
\text { cristalino }\end{array}$ \\
\hline 3 & $\begin{array}{c}\text { Picante, ácido, ligeramente } \\
\text { dulce }\end{array}$ & Incoloro cristalino & $\begin{array}{c}\text { Intensamente aromático } \\
\text { a anís, agradable. }\end{array}$ & - \\
\hline 4 & Dulce, ligeramente ácido & - & - & - \\
\hline 5 & $\begin{array}{l}\text { Muy dulce, aromático y } \\
\text { ligeramente ácido }\end{array}$ & - & - & - \\
\hline
\end{tabular}

Fuente: Elaboración propia. 


\section{Análisis fisicoquímico}

Se midieron las variables siguiendo métodos, procedimientos, lineamientos y/o especificaciones establecidas y reguladas.

- Grado alcohólico: Se midió según la norma NTE INEN 3409.

- Densidad: Se midió según la norma NTP ISO 279: $2011^{10}$.

- $\quad$ Acidez: Se midió según la norma NTP 211.027:2003 ${ }^{11}$.

- $\quad$ Punto de ebullición: Se midió según el método semi micro.

- Índice de refracción: Se midió según la norma NTP ISO 280:20112.

- IÍndice de esteres: Se midió según la norma NTP 319-088 INDECOPI ${ }^{13}$.

- $\quad$ Índice de carbonilos: Se midió según la norma NTP 319.078:19744.

\section{Análisis espectroscópicos y cromatográficos}

\subsection{Espectroscopia UV-visible}

El equipo que se utilizó para la determinación de la longitud de onda de los estándares (trans-Anetol y p-Anisaldehído) fue un espectrofotómetro UV-visible modelo GENESYS 10S con software Vision lite, lámpara de xenón y rango de longitud de onda de 190 a 1100 $\mathrm{nm}$. Se realizó un barrido espectral a un rango de 200-400 nm; para lo cual se preparó una solución de $0,1 \mu \mathrm{L}$ de trans-Anetol $/ 10 \mathrm{~mL}$ de etanol p.a. y $0,1 \mu \mathrm{L}$ de $p$-Anisaldehído/10 $\mathrm{mL}$ en etanol p.a.

\subsubsection{Determinación de la curva de calibración}

Para el caso del trans-Anetol, la curva de calibración se realizó con etanol p.a. y estándar de trans-Anetol. Se preparó una solución madre de $12,3 \mathrm{mg} / \mathrm{L}$ en $100 \mathrm{~mL}$ y después se realizaron diluciones. A partir de la curva se obtuvo la ecuación de regresión lineal $\mathrm{Y}=$ $0,4607 x$, con un $\mathrm{R}=0,9918$. Para el caso del $p$-Anisaldehído, la curva de calibración se realizó con etanol p.a. y estándar de $p$-Anisaldehído. Se preparó una solución madre de $12,3 \mathrm{mg} / \mathrm{L}$, en $100 \mathrm{~mL}$ y después se realizaron diluciones. A partir de la curva se obtuvo la ecuación de regresión lineal $\mathrm{Y}=0,0499 \mathrm{x}-0,0044$, con un $\mathrm{R}=0,9991$. Las muestras de destilado fueron centrifugadas, filtradas y diluidas $10 \mu \mathrm{L}$ en una fiola de $10 \mathrm{~mL}$.

\subsection{Cromatografía en Capa Fina (CCF)}

Mediante esta técnica se determina cualitativamente la presencia de compuestos mayoritarios. Se inició con la preparación de la cámara cromatográfica; se colocó en la cámara acetato de etilo: tolueno (7:93), se homogenizó por agitación y se dejó reposar por 10 minutos. En las cromatoplacas de Silicagel $60 \mathrm{~F}_{254}$, con soporte de vidrio de $20 \times 20$ $\mathrm{cm}$, adquiridas en Merck Perú; se trazó una línea de base a una distancia de 1,5 cm por encima de la base de la placa y luego se realizó la activación en una estufa a $60{ }^{\circ} \mathrm{C}$ por un periodo de 1 hora. Posteriormente se realizó el sembrado de las muestras, extrayendo $5 \mu \mathrm{L}$ y colocándolos por encima de la línea de base, a continuación se introdujo en la cámara que debe ser cerrada herméticamente. Cada 5 minutos fue verificado que el solvente no rebase la línea marcada en la cromatoplaca $(\mathrm{L}=1,5 \mathrm{~cm})$. Se utilizó como fase estacionaria metanol, y para la revelación se utilizó revelador $\mathrm{H}_{2} \mathrm{SO}_{4}$ : Vainillina y Lámpara UV de 254 nm. 


\subsection{Cromatografía Líquida de Alta Eficiencia (CLAE)}

Las corridas se realizaron en un cromatógrafo tipo LACHROM LITE - CLAE, con bomba cuaternaria de baja presión, detector de diodos, espectrofotómetro UV-visible, automuestreador y una columna RP C18 de $125 \mathrm{~mm}$. Se utilizaron como fase móvil y estacionaria metanol grado CLAE y una columna RP C18 $125 \mathrm{~mm}$, con una velocidad de flujo de $1 \mathrm{~mL} / \mathrm{min}$, una temperatura de columna de $30 \pm 1{ }^{\circ} \mathrm{C}$, y un volumen de inyección de muestra de $20 \mu \mathrm{L}$. Los estándares y las muestras fueron filtrados en una membrana fina de $0,45 \mu \mathrm{m}$ para llevar a cabo una corrida de cada uno de ellos a $265 \mathrm{~nm}$, obteniendo un tiempo de retención de 12,440 min para el trans-Anetol y de 2,873 min para el p-Anisaldehído. Las muestras fueron preparadas en una solución de metanol:agua ultra pura (50:50) y se realizaron las corridas por triplicado. Esta técnica se utilizó para determinar la concentración de los componentes mayoritarios (trans-Anetol y p-Anisaldehído) en las muestras, utilizando como referencia los estándares correspondientes a una concentración de 99,99\%. Este análisis fue realizado por un Laboratorio de Control de Calidad Acreditado por INACAL.

\subsection{Cromatografía de Gases Acoplada a Espectrometría de Masas (CG/EM)}

El equipo que se utilizó fue un cromatógrafo SHIMADZU GC-2010, versión 2100; con detector de ionización a la llama y espectroscopía de masas, con columna capilar SPD 20, inyector automático. La corrida se realizó en las siguientes condiciones: temperatura de columna $45 \pm 3^{\circ} \mathrm{C}$, temperatura de inyección de $250^{\circ} \mathrm{C}$, temperatura de detección de $300^{\circ} \mathrm{C}$, y fase estacionaria Helio. Las muestras fueron preparadas en una solución de Hexano $(5 \mu \mathrm{L} / 10 \mathrm{~mL}$ de solvente) y luego fueron filtradas. Este análisis fue realizado por un laboratorio de control de calidad acreditado por INACAL.

\section{RESULTADOS Y DISCUSIÓN}

\section{Análisis organoléptico}

En la tabla 3 se muestran los resultados del análisis organoléptico realizado a las muestras de destilado alcohólico de anís verde obtenidas con uno (01), dos (02) y tres (03) platos.

Tabla 3. Resultados del análisis organoléptico.

\begin{tabular}{|c|c|c|c|}
\hline Variable & 01 plato & 02 platos & 03 platos \\
\hline Sabor & $\begin{array}{l}\text { Picante, ácido, } \\
\text { ligeramente dulce (3) }\end{array}$ & $\begin{array}{l}\text { Muy dulce, aromático y } \\
\text { ligeramente ácido (5) }\end{array}$ & $\begin{array}{c}\text { Dulce, } \\
\text { ligeramente } \\
\text { ácido (4) }\end{array}$ \\
\hline Color & $\begin{array}{l}\text { Incoloro, ligeramente } \\
\text { amarillento ( } 2)\end{array}$ & Incoloro cristalino (3) & $\begin{array}{c}\text { Incoloro } \\
\text { cristalino (3) }\end{array}$ \\
\hline Olor & $\begin{array}{l}\text { Ligeramente } \\
\text { aromático a anís y } \\
\text { penetrante (2) }\end{array}$ & $\begin{array}{l}\text { Intensamente aromático a } \\
\text { anís, agradable (3) }\end{array}$ & $\begin{array}{c}\text { Ligeramente } \\
\text { aromático a } \\
\text { anís, agradable } \\
(2.5)\end{array}$ \\
\hline Estado físico & $\begin{array}{l}\text { Líquido cristalino } \\
\text { ligeramente } \\
\text { opalescente }(1,5)\end{array}$ & Líquido cristalino (2) & $\begin{array}{c}\text { Líquido } \\
\text { cristalino (2) }\end{array}$ \\
\hline
\end{tabular}

Fuente: Elaboración propia. 
El sabor y el olor son las características más importantes para valorar la calidad del destilado y diferenciarlos. Los resultados confieren al destilado obtenido con dos (02) platos las mejores características organolépticas para ser utilizado como insumo principal en la elaboración del producto final al cual está destinado.

\section{Análisis fisicoquímicos}

En la tabla 4 se muestran los resultados de los análisis fisicoquímicos realizados a las muestras de destilado alcohólico de anís verde obtenidas con uno (01), dos (02) y tres (03) platos.

Tabla 4. Resultados de los análisis fisicoquímicos.

\begin{tabular}{cccc}
\hline Variable & 01 plato & 02 platos & 03 platos \\
\hline Grado alcohólico & 61,00 & 61,23 & 60,08 \\
Acidez & 2,541 & 3,217 & 3,415 \\
Densidad & 0,9099 & 0,9091 & 0,9119 \\
Índice de refracción & 1,363 & 1,362 & 1,363 \\
Punto de ebullición & 83,2 & 81,2 & 81,2 \\
Índice de éster & 8,31 & 7,62 & 6,78 \\
Índice de carbonilos & 8,22 & 15,15 & 22,12 \\
\hline
\end{tabular}

Fuente: Elaboración propia.

El destilado obtenido con dos (02) platos tiene $61,23^{\circ} \mathrm{GL}$, un mayor grado alcohólico que el obtenido con uno (01) y tres (03) platos, lo que indica que se da una mejor separación y arrastre de compuestos aromáticos. Respecto a la acidez, el destilado obtenido con un plato presenta menor acidez, sin embargo, esta se ve afectada con el paso del tiempo debido a la oxidación de alcoholes, aldehídos e hidrólisis de los ésteres presentes. En el caso de la densidad, el destilado obtenido con tres (03) platos tiene mayor densidad, es decir, mayor cantidad de extractos (sustancias no volátiles) que los otros destilados.

El índice de refracción (IR) es una variable que guarda relación con la composición química: si hay predominancia de compuestos bencénicos el IR es mayor a 1,50 y si no la hay, el IR es próximo a 1,45. Los índices de refracción de destilados obtenidos con uno (1), dos (02) y tres (3) platos son similares, con predominancia de compuestos no aromáticos. Respecto al índice de éster, es menor en los destilados obtenidos con dos (02) y tres (3) platos, lo que indica la presencia de ésteres de baja masa molecular. El índice de carbonilos del destilado de tres (03) platos es $22,12 \%$, mientras que de los destilados de un (01) plato y dos (02) platos son 8,22 y $15,15 \%$, respectivamente; es decir, el de tres (03) platos tiene mayor presencia de aldehídos y cetonas.

\section{Análisis cromatográficos y espectroscópicos}

\subsection{Espectroscopia UV-visible}

En las tablas 5 y 6 se muestran los resultados de los análisis de Espectroscopia UV-visible realizados a las muestras de destilado alcohólico de anís verde obtenidas con uno (01), dos (02) y tres (03) platos. 
Tabla 5. Resultados de los análisis de Espectroscopia UV-visible: concentración de trans-Anetol (mg/L).

\begin{tabular}{cccc}
\hline Prueba & 01 plato & 02 platos & 03 platos \\
\hline P1 (mg/L) & 1,09 & 1,02 & 1,45 \\
P2 $(\mathbf{m g} / \mathbf{L})$ & 1,08 & 1,16 & 1,35 \\
P3 (mg/L) & 1,12 & 1,09 & 1,26 \\
P4 (mg/L) & 1,08 & 1,09 & 1,26 \\
P5 (mg/L) & 0,95 & 1,16 & 1,36 \\
\hline
\end{tabular}

Fuente: Elaboración propia.

El destilado obtenido con tres (03) platos tiene mayor concentración de trans-Anetol que los obtenidos con uno (01) y dos (02) platos.

Tabla 6. Resultados de los análisis de Espectroscopia UV-visible: concentración de p-Anisaldehído (mg/L).

\begin{tabular}{cccc}
\hline Prueba & 01 plato & 02 platos & 03 platos \\
\hline P1 (mg/L) & 5,02 & 4,26 & 4,86 \\
P2 (mg/L) & 5,82 & 4,12 & 4,76 \\
P3 (mg/L) & 4,74 & 4,90 & 4,72 \\
P4 (mg/L) & 5,12 & 4,58 & 4,72 \\
P5 (mg/L) & 5,74 & 4,64 & 5,82 \\
\hline
\end{tabular}

Fuente: Elaboración propia.

El destilado obtenido con dos (02) platos presenta una menor concentración de $p$-Anisaldehído, motivo por el cual existe una menor probabilidad de incrementar su acidez con el tiempo; es decir, es más estable.

\subsection{Cromatografía en Capa Fina}

En la figura 1 y en la tabla 7 se muestran los resultados de la cromatografía en capa fina realizada a las muestras de destilado alcohólico de anís verde obtenidas con uno (01), dos (02) y tres (03) platos. 


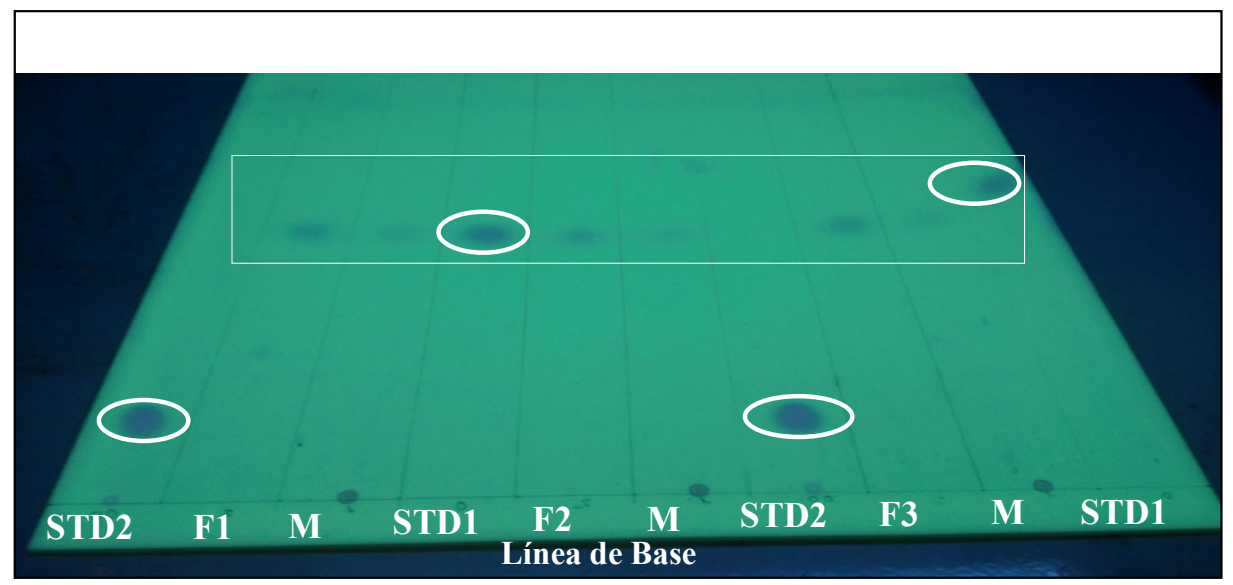

Figura 1. Resultados de los análisis de Cromatografía en Capa Fina.

Leyenda: STD1: Estándar de trans-Anetol; STD2: Estándar de $p$-Anisaldehído; M: Macerado; F1: Destilado de un (01) plato; F2: Destilado de dos (02) platos y F3: Destilado de tres (03) platos.

Tabla 7. Resultado de los análisis de Cromatografía en Capa Fina (Rf.).

\begin{tabular}{cccccc}
\hline Fase móvil & \multicolumn{2}{c}{ Estándar } & 01 plato & 02 platos & 03 platos \\
\hline Tolueno: Acetato & trans-Anetol & 0,86 & 0,85 & 0,85 & 0,84 \\
de Etilo (7:93) & $p$-Anisaldehído & 0,50 & 0,00 & 0,00 & 0,00 \\
\hline
\end{tabular}

Fuente: Elaboración propia.

La tabla 7 y la figura 1 muestran la relación de frente que se obtuvo de los destilados, las cuales se compararon con la relación de frente de los estándares STD1 (trans-Anetol) y STD2 ( $p$-Anisaldehído) indicando la presencia de trans-Anetol y la ausencia de $p$-Anisaldehído.

\subsection{Cromatografía Líquida de Alta Eficiencia (CLAE)}

La figura 2 muestra los cromatogramas de las muestras de los estándares del $p$-Anisaldehído y del trans-Anetol, mientras que en la tabla 8 se presentan los resultados de los análisis de CLAE realizados a las muestras de destilado alcohólico de anís verde obtenidas con uno (01), dos (02) y tres (03) platos. 


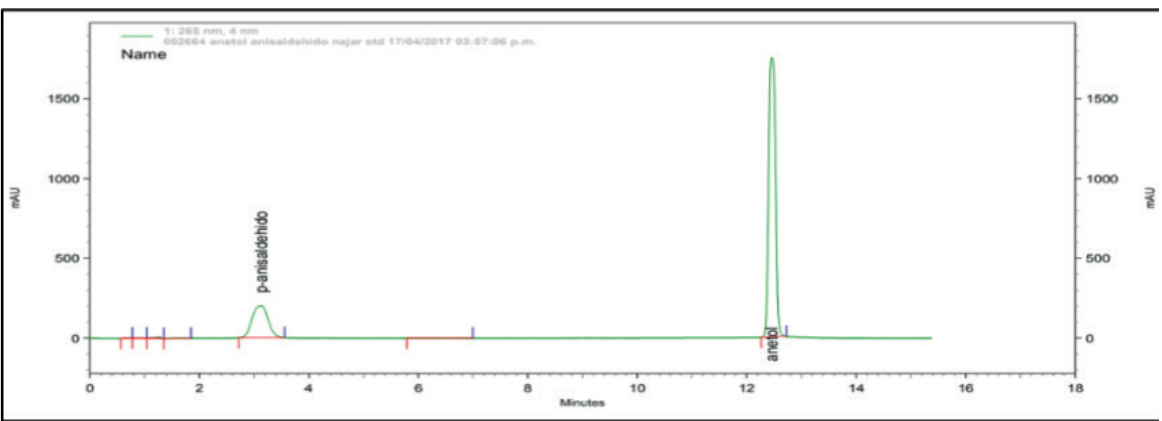

Figura 2. Cromatograma del p-Anisaldehído y del trans-Anetol obtenido en los análisis de CLAE.

Tabla 8. Resultados de los análisis de CLAE.

\begin{tabular}{cccc}
\hline Compuesto & 01 plato & 02 platos & 03 platos \\
\hline trans-Anetol & 0,1164 & 0,1169 & 0,1057 \\
$\boldsymbol{p}$-Anisaldehído & No detectable & No detectable & No detectable \\
\hline
\end{tabular}

Fuente: Elaboración propia.

El destilado obtenido con dos (02) platos tiene 0,1169\% de trans-Anetol, seguido por el obtenido con un (01) plato con $0,1164 \%$ y el obtenido con tres $(03)$ platos con $0,1057 \%$. No se detectó $p$-Anisaldehído en ninguna de las muestras.

\subsection{Cromatografía de Gases Acoplada a Espectrometría de Masas (CG/EM)}

En las figuras 3, 4 y 5 se pueden observar los cromatogramas obtenidos para las muestras de destilado alcohólico de anís verde obtenidas con uno (01), dos (02) y tres (03) platos, respectivamente; y en la tabla 9 se muestran los resultados de los análisis de GC/EM realizados a las muestras mencionadas.

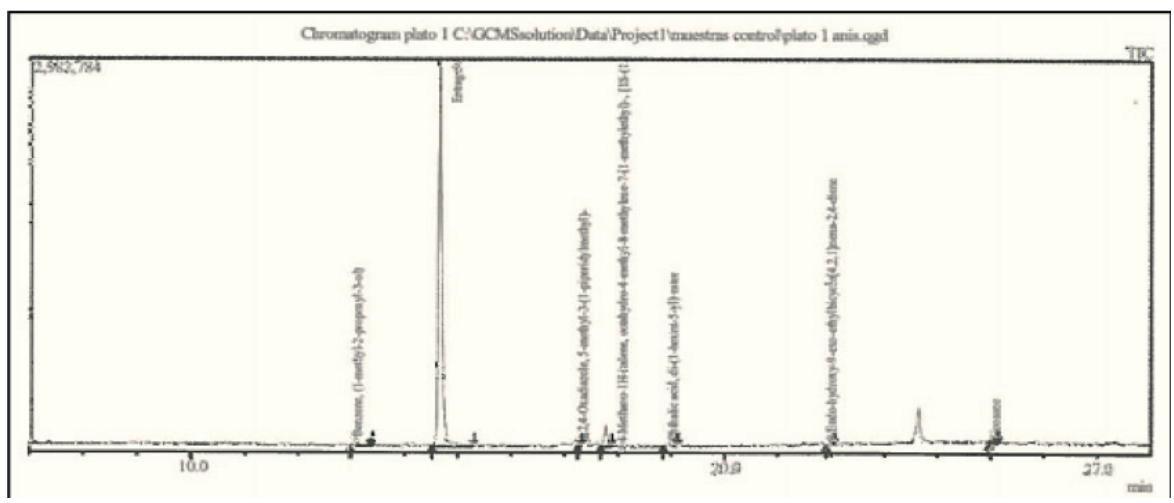

Figura 3. Cromatograma del destilado obtenido con un (01) plato. 


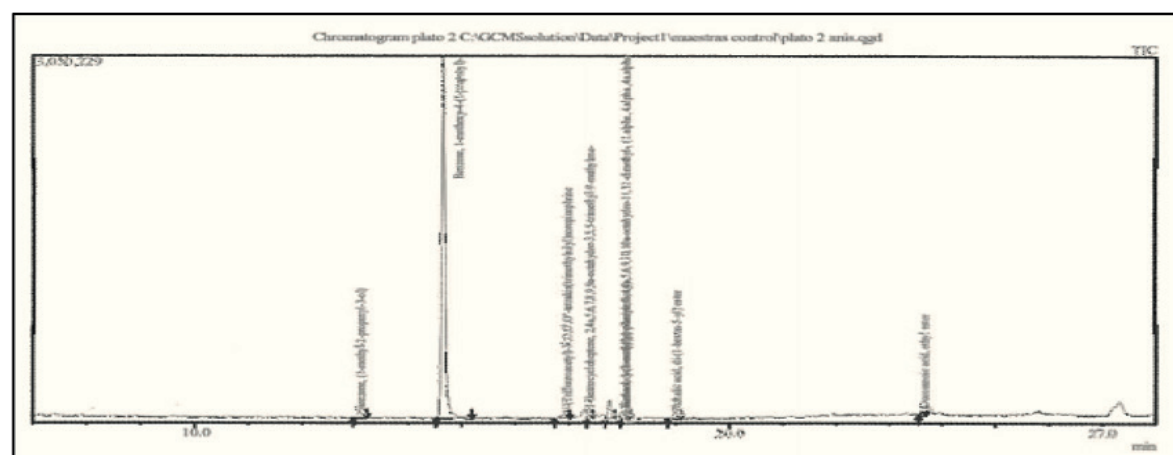

Figura 4. Cromatograma del destilado obtenido con dos (02) platos.

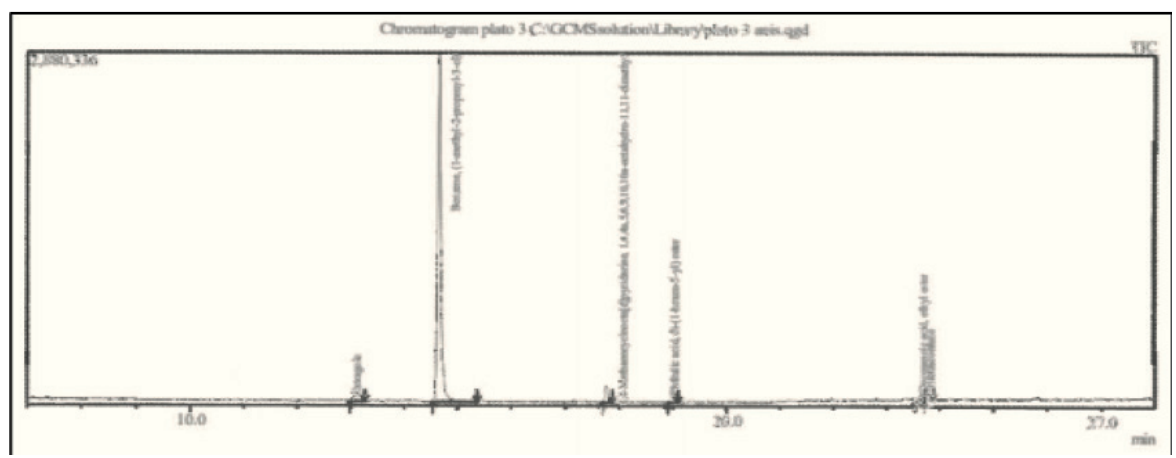

Figura 5. Cromatograma del destilado obtenido con tres (03) platos. 
Tabla 7. Resultado de los análisis de Cromatografía en Capa Fina (Rf.).

\begin{tabular}{|c|c|c|c|c|c|c|}
\hline \multirow[b]{2}{*}{ Compuesto } & \multicolumn{2}{|c|}{01 plato } & \multicolumn{2}{|c|}{02 platos } & \multicolumn{2}{|c|}{03 platos } \\
\hline & $\begin{array}{l}\text { Tiempo de } \\
\text { retención } \\
\text { (min) }\end{array}$ & $\begin{array}{c}\text { Área } \\
(\%)\end{array}$ & $\begin{array}{l}\text { Tiempo de } \\
\text { retención } \\
(\mathrm{min})\end{array}$ & $\begin{array}{l}\text { Área } \\
(\%)\end{array}$ & $\begin{array}{l}\text { Tiempo de } \\
\text { retención } \\
\text { (min) }\end{array}$ & $\begin{array}{l}\text { Área } \\
(\%)\end{array}$ \\
\hline trans-Anetol & 14,62 & 90,15 & 14,63 & 86,83 & 14,63 & 88,16 \\
\hline $\begin{array}{l}\text { 1,4-metano-1H-indeno, } \\
\text { octahidro-4-metil }\end{array}$ & 17,75 & 3,88 & - & - & & - \\
\hline Estragol & 13,05 & 2,09 & 13,05 & 1,75 & 13,06 & 1,67 \\
\hline $\begin{array}{l}\text { Acido ftálico, di-(1- } \\
\text { hexen-5-il) éster }\end{array}$ & 18,98 & 1,77 & 18,98 & 1,84 & 18,98 & 2,08 \\
\hline $\begin{array}{l}\text { Oxadiazol, metil-3-(1- } \\
\text { piperidil) }\end{array}$ & 17,29 & 0,71 & - & - & - & - \\
\hline $\begin{array}{c}\text { 1,4-metanocicloocta }[\mathrm{d}] \\
\text { piridacina }\end{array}$ & - & - & 17,75 & 3,88 & 17,75 & 3,46 \\
\hline $\begin{array}{c}\text { 2-butanol, 3-(1-metil-2- } \\
\text { fenil) }\end{array}$ & - & - & 18,02 & 0,89 & & - \\
\hline Etil éster, ácido & - & - & 23,63 & 2,14 & 23,64 & 2,53 \\
\hline $\begin{array}{c}\text { N-(trifluoroacetil)-N-o, } \\
\text { o }^{\prime}, \text { o' }^{\prime} \text { - tris } \\
\text { (trimetilsilil) }\end{array}$ & - & - & 16,93 & 1,99 & & - \\
\hline$\alpha$-Himachalene & - & - & 17,35 & 0,68 & & - \\
\hline Eicosano & 25,04 & 0,69 & - & - & & - \\
\hline Tritetracontano & - & - & - & - & 23,77 & 2,1 \\
\hline $\begin{array}{c}\text { 9-endo-hidroxi-9-exo- } \\
\text { etil-biciclo-[4.2.1]-nona- } \\
2,4-\text {-dieno }\end{array}$ & 21,96 & 0,71 & - & - & & - \\
\hline
\end{tabular}

Fuente: Elaboración propia.

En el destilado obtenido con dos (02) platos se identificaron ocho componentes predominando el trans-Anetol con $86,83 \%$, en los destilados con uno (01) y tres (03) platos se identificaron siete y seis componentes predominando el trans-Anetol con 90,15\% y 88,16 $\%$, respectivamente. En los tres destilados se tiene un tiempo de retención de 14,62 a 14,63 min del compuesto mayoritario.

\section{CONCLUSIONES}

En general, los destilados obtenidos presentan compuestos carbonilos, insaturados y carbohidratos, siendo los destilados con dos (02) y tres (03) platos los que presentan el mejor sabor y olor. Los análisis de UV-VIS, CCF, CLAE y CG/EM revelan que el compuesto mayoritario en los destilados es el trans-Anetol. Según los resultados de los análisis realizados y los requerimientos del anisado (bebida alcohólica obtenida por aromatización de alcohol con variedades de anís) producido por la industria licorera donde se realizó el trabajo, se concluye que el destilado de dos (02) platos presenta mejores características y presencia de componentes que lo hacen diferente y mejor que los demás para la formulación del producto final. 


\section{AGRADECIMIENTOS}

Agradecemos a INNÓVATE PERÚ por haber cofinanciado junto con la empresa Manuel Muñoz Najar S.A.C. Industria Licorera el proyecto Contrato $N^{\circ}$ 031-PITEI-2016, a partir del cual se realizó el presente trabajo. Agradecemos a todos nuestros familiares, amigos y compañeros de trabajo por el apoyo brindado.

\section{REFERENCIAS BIBLIOGRÁFICAS}

1. European pharmacopoeia. Monograph on anise fruit oil. [Internet] 9na ed. España; 2002. [citado 12 mar 2019]. Disponible en: https://www.edqm.eu/en/european-pharmacopoeia9th-edition

2. Orav A, Raal A. Essential oil composition of P. anisum L. fruits from various European countries. Nat Prod Res. 2008; 22: 227-232.

3. Chaquilla G, Estela W, Torres V, Ballinas M, Gastélum M, Nevárez V. Composición química y contenido de fenoles totales en aceite esencial de muña Minthostachys setosa Briq Epl y anís P. anisum L. ECIPERU. 2012; 8 (2): 107-111.

4. Trigo I, De Groot W, Medrano N, Espinoza J. Extracción y caracterización del aceite esencial P. anisum L., producido en Bolivia. Agron Colomb. 2016, 34 (1): S1447-S1449.

5. Quiñones Y. Análisis de los principios activos de especies mexicanas de anís y evaluación de su actividad biológica sobre microorganismos patógenos. [Tesis Doctoral]. Nuevo León, México: Universidad Autónoma de Nuevo León; 2011.

6. Norma Técnica Peruana NTP 319.079.1974. Aceites esenciales. Extracción de muestras. Lima: INDECOPI; 2016.

7. Norma Técnica Peruana NTP-ISO 11035. Análisis sensorial. Metodología. Lima: INDECOPI; 2009.

8. Norma Técnica Chilena NCh-107. Análisis sensorial. Aceites. Chile: INN; 2001.

9. Norma Técnica Ecuatoriana NTE INEN 340. Bebidas alcohólicas. Determinación del grado alcohólico.

10. Norma Técnica Peruana NTP ISO 279: 2011. Determinación de la densidad. Lima: INDECOPI; 2016.

11. Norma Técnica Peruana NTP 211.027. Bebidas Alcohólicas. Alcohol etílico. Método de ensayo. Determinación de la acidez total. Lima: INDECOPI; 2003.

12. Norma Técnica Peruana NTP ISO 280. Determinación del Índice de Refracción. Lima: INDECOPI; 2016.

13. Norma Técnica Peruana NTP 319.088. Determinación de índice de esteres. Lima: INDECOPI; 2016.

14. Norma Técnica Peruana NTP 319.078. Determinación de índice de carbonilos. Lima: INDECOPI; 2016. 